\title{
Pulse duration and wavelength stability measurements of a midinfrared free-electron laser
}

\section{$\operatorname{AUTHOR}(\mathrm{S})$ :}

Qin, Yu; Zen, Heishun; Wang, Xiaolong; Kii, Toshiteru; Nakajima, Takashi; Ohgaki, Hideaki

\section{CITATION:}

Qin, Yu ...[et al]. Pulse duration and wavelength stability measurements of a midinfrared free-electron laser. Optics Letters 2013, 38(7): 10681070

\section{ISSUE DATE:}

2013-04

URL:

http://hdl.handle.net/2433/173323

\section{RIGHT:}

(c) 2013 Optical Society of America.; This paper was published in "Optics Letters" and is made available as an electronic reprint with the permission of OSA. The paper can be found at the following URL on the OSA website:

http://www.opticsinfobase.org/ol/abstract.cfm?uri=ol-38-7-1068. Systematic or multiple reproduction or distribution to multiple locations via electronic or other means is prohibited and is subject to penalties under law. 


\title{
Pulse duration and wavelength stability measurements of a midinfrared free-electron laser
}

\author{
Yu Qin, ${ }^{1}$ Heishun Zen, ${ }^{1}$ Xiaolong Wang, ${ }^{1}$ Toshiteru Kii, ${ }^{1}$ Takashi Nakajima, ${ }^{1, *}$ and Hideaki Ohgaki ${ }^{1,2}$ \\ ${ }^{1}$ Institute of Advanced Energy, Kyoto University, Gokasho, Uji, Kyoto 611-0011, Japan \\ ${ }^{2}$ e-mail: ohgaki@iae.kyoto-u.ac.jp \\ ${ }^{*}$ Corresponding author: t-nakajima@iae.kyoto-u.ac.jp
}

Received December 28, 2012; revised February 18, 2013; accepted March 2, 2013;

posted March 4, 2013 (Doc. ID 182477); published March 21, 2013

\begin{abstract}
We report the pulse duration and wavelength stability measurements of a midinfrared free-electron laser (FEL) where the wavelength fluctuation may not be negligible. The technique we employ is a fringe-resolved autocorrelation (FRAC) method that has good sensitivity on not only the pulse duration and the chirp but also the wavelength stability. By the simple manipulation of experimental FRAC signals, we can obtain the pulse duration even if the amounts of the chirp and the wavelength stability are not known in advance, which is further used to estimate the wavelength stability. Through this procedure we find that the pulse duration of the Kyoto University FEL at $12 \mu \mathrm{m}$ is about $0.58 \mathrm{ps}$ without any notable chirp, and the wavelength stability is about $1.3 \%$. We also carry out separate experiments for intensity autocorrelation and sum-frequency mixing. The difference we find for pulse duration and wavelength stability by the different measurements is attributed to the different operation conditions of FEL. (c) 2013 Optical Society of America
\end{abstract}

OCIS codes: $\quad 140.2600,040.3060,320.7110,190.7220$.

Unlike conventional optical lasers, there is no fundamental limitation for the working wavelength of free-electron lasers (FELs). As a result, at the wavelength range where conventional optical lasers do not work, FELs are the only choice as a powerful coherent light source, and have attracted a lot of interest in recent years [1].

The FEL we have at Kyoto University (KU-FEL) is an oscillator-type FEL at the midinfrared (mid-IR) wavelength $(5-13 \mu \mathrm{m})$ [2]. A bright coherent light source in this wavelength region is very useful for various purposes such as those involving resonant transitions between vibrational states of molecules [3] . Apart from the obvious importance of the absolute wavelength stability of FELs for spectroscopic applications, quantifying the relative wavelength stability itself is an important issue to assess the performance of FELs. However, realization of good wavelength stability of FELs is not an easy task. This can be easily understood if one recalls that the FEL pulses originate from the accelerated electron bunches, and their energy and phase are very difficult to maintain constant. Furthermore, the lasing of FELs is triggered by spontaneous emissions that may have slightly different photon energies within the allowed cavity modes. As a result there may be a micropulse-to-micropulse change in the FEL wavelength even within the same macropulse. Accordingly a single-macropulse measurement of the FEL spectrum using an array-type detector for the mid-IR does not help to estimate the wavelength stability.

In this Letter, we report the measurements of the pulse duration and wavelength stability of KU-FEL by the fringe-resolved autocorrelation (FRAC) method [4-7]. Note that the FRAC signals are influenced by not only the pulse duration and the chirp, which is well-known, but also the wavelength stability, which was first discussed, to our knowledge, in our previous paper [8]. Under the presence of unknown amounts of chirp and wavelength stability, extracting the pulse duration from FRAC signals is not a very trivial issue for a low repetition laser such as the KU-FEL. We first show that a simple manipulation of the experimental FRAC data gives us the pulse duration with reasonably good accuracy. Then, using the pulse duration we obtain, we estimate the wavelength stability. This is an improved version of the method we have proposed in a recent paper [8], where we have assumed that the pulse duration is known in advance by the separate intensity autocorrelation (IAC) measurement.

Before proceeding further, we briefly explain why we can use the FRAC method to estimate the wavelength stability of a laser. This can be most easily understood if we recall that the modulation period in the FRAC signals directly reflects the optical period of the incident laser wavelength. For instance, if there are two frequency components in the incident laser, there are two modulation periods in the FRAC signals. Clearly if the FRAC signals with two different modulation periods are superposed, the modulation in the summed FRAC signals dies away quickly as the delay becomes large, and the larger the frequency differences, the narrower the FRAC signal

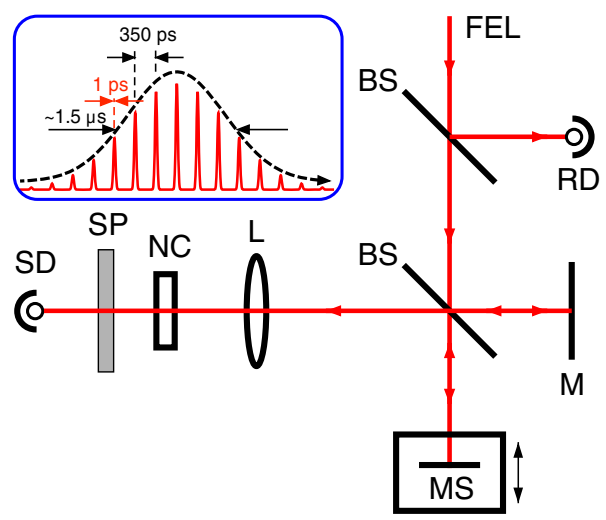

Fig. 1. (Color online) Experimental setup for the FRAC measurement. BS, M, L, NC, and SP represent the beam splitter, mirror, focusing lens, nonlinear crystal, and short-pass filter. MS, $\mathrm{RD}$, and SD stand for the motorized stage, reference signal detector, and signal detector, respectively. The inset shows the pulse structure of the KU-FEL. 
width becomes. See Fig. 1 of [8]. This implies that we can correlate the narrowing of the FRAC signal width with the wavelength stability [8]. Actually the above argument is very similar to that explaining why the FRAC signal width of chirped pulses appears narrower than that of transform-limited pulses with the same pulse duration.

The experimental setup for the FRAC measurement is shown in Fig. 1. A KU-FEL pulse (macropulse duration $\sim 1.5 \mu \mathrm{s}$ at a repetition rate of $1 \mathrm{~Hz}$ and micropulse duration $\sim 1$ ps with a time interval of $350 \mathrm{ps}$ ) tuned to $12 \mu \mathrm{m}$ is split into two by a beam splitter (Edmund, NT62-967) to form a Michelson interferometer. After a variable time delay introduced by the motorized stage (Sigma Koki, PAT-001) with a step size of $2 \mu \mathrm{m}$ (round trip), the two pulses in both arms are combined coherently by the same beam splitter, and then focused with an $f=200 \mathrm{~mm} \mathrm{ZnSe}$ lens to the $\mathrm{AgGaSe}_{2}$ crystal (EKSMA, type I, $1 \mathrm{~mm}$ thickness, 67.7 deg cut angle) to generate the second harmonic (SH). After a short-pass filter (Spectrogon, SP-7150), the SH energy is measured by a signal detector (Gentec. EO, QE8SP-I-BL-BNC), which we call $E_{\text {sig. }}$. For the appropriate normalization of the SH signals, we monitor the macropulse energy with a reference signal detector (Gentec. EO, QE8SP-I-BL-BNC), which we call $E_{\text {ref }}$. We record $S_{\text {FRAC }}=$ $E_{\text {sig }} / E_{\text {ref }}^{2}$ at different delays as FRAC signals after averaging over three macropulses for each delay.

In Fig. 2(a) we present the FRAC signals, where the red lines show the upper and lower envelopes of the FRAC signals for a guide to the eye. The blow-up of the FRAC signals near delay zero is presented in Fig. 2(b), which clearly shows a modulation period of $40 \mathrm{fs}$, corresponding to the optical cycle of the $12 \mu \mathrm{m}$ laser.

The first question we must solve is, "How do we obtain the pulse duration from the FRAC signals when the amounts of chirp and wavelength stability are not known?" In the following, we show that we can obtain the pulse duration, regardless of the presence of the unknown amounts of chirp and wavelength stability, only from the FRAC signals. We would like to emphasize that this is not as obvious as it sounds, because the width of the FRAC signals would be significantly narrowed by the presence of chirp and wavelength stability []ㅡ, both of which are unknown.

To extract the pulse duration from the FRAC signals, we assume that each micropulse has a Gaussian temporal shape with a linear chirp. After the appropriate
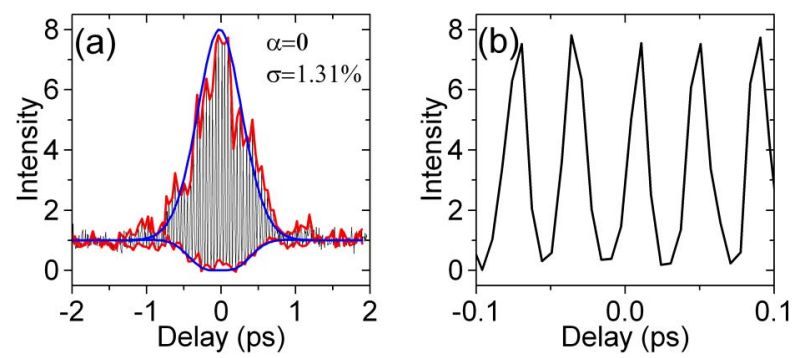

Fig. 2. (Color online) FRAC signals of KU-FEL at $12 \mu \mathrm{m}$. (a) Entire signals and (b) blow-up signals around delay zero. In graph (a) the rapidly oscillating lines and zig-zag lines represent the measured FRAC signals and its upper and lower envelopes, respectively, while the slowly varying lines represent the fitted envelopes. The fitted values are $\alpha=0 \%$ and $\sigma=1.31 \%$. normalization we define the electric field of the $k$ th micropulse as

$$
\begin{aligned}
E^{(k)}(t)= & \exp \left[-2\left(\frac{t}{\tau_{0}}\right)^{2} \frac{1}{1-i \alpha} \ln 2\right] \\
& \times \exp \left[i\left(a^{(k)} \omega_{0} t+\phi^{(k)}(t)\right)\right],
\end{aligned}
$$

where $\tau_{0}, \omega_{0}$, and $\alpha$ are the transform-limited pulse duration for the corresponding spectral width, the central frequency, and the dimensionless chirp parameter of micropulses. Provided with $\tau_{0}$ and $\alpha$, the micropulse duration, $\tau_{p}$, defined for the full width at half-maximum (FWHM), is given by $\tau_{p}=\tau_{0} \sqrt{1+\alpha^{2}} . \phi^{(k)}(t)$ is the phase that is set to be 0 in this Letter, and $a^{(k)} \omega_{0}$ represents the carrier frequency, which may be a bit different from $\omega_{0}$. That is, introducing a normal distribution function, $N$, and its variance, $\sigma, a^{(k)}$ is written as $a^{(k)} \sim N\left(1, \sigma^{2}\right)$, where the first argument, 1, stands for the average value of $a^{(k)}$. After some algebra, the expectation value of the FRAC signals, $\left\langle S_{\text {FRAC }}(\tau)\right\rangle$, as a function of the delay time between the two pulses, $\tau$, is found to be

$$
\begin{aligned}
\left\langle S_{\mathrm{FRAC}}(\tau)\right\rangle= & \left\langle\int_{-\infty}^{\infty}[E(t)+E(t-\tau)]^{4} \mathrm{~d} t\right\rangle \\
= & 1+4 \exp \left[\frac{-\left(3+\alpha^{2}\right) \tau^{2} \ln 2}{2 \tau_{p}^{2}}-\frac{\sigma^{2} \omega_{0}^{2} \tau^{2}}{2}\right] \\
& \times \cos \left(\frac{\alpha^{2} \tau^{2} \ln 2}{2 \tau_{p}^{2}}\right) \cos \left(\omega_{0} \tau\right)+2 \exp \left(\frac{-2 \tau^{2} \ln 2}{\tau_{p}^{2}}\right) \\
& +\exp \left(\frac{-2\left(1+\alpha^{2}\right) \tau^{2} \ln 2}{\tau_{p}^{2}}-2 \sigma^{2} \omega_{0}^{2} \tau^{2}\right) \cos \left(2 \omega_{0} \tau\right),
\end{aligned}
$$

from which the upper and lower envelopes of $\left\langle S_{\text {FRAC }}(\tau)\right\rangle$ can be easily obtained by replacing $\omega_{0} \tau$ by 0 or $\pi$. Note that the quantities $\sigma$ and $\alpha$, which represent the wavelength stability and the chirp, respectively, are unknown in Eq. (2), and our question is how to extract $\tau_{p}$ under such situations.

By noting that the second and fourth terms in Eq. (2) are rapidly oscillating, we carry out the integration over $\tau$ after subtracting the constant term, 1 , and obtain

$$
\int_{-\infty}^{\infty}\left\langle S_{\mathrm{FRAC}}(\tau)-1\right\rangle \mathrm{d} \tau=\sqrt{\frac{2 \pi}{\ln 2}} \tau_{p},
$$

which is nothing but the time integration of the third term of Eq. (2), and proportional to the pulse duration, $\tau_{p}$. It is very important to note that Eq. (3) does not contain the unknown quantities $\sigma$ and $\alpha$ anymore. Furthermore, by performing the summation to approximate the integration, we finally obtain

$$
\tau_{p}=\sqrt{\frac{\ln 2}{2 \pi}} \Delta \tau \sum_{i=1}^{n}\left\langle S_{\mathrm{FRAC}}\left(\tau_{i}\right)-1\right\rangle,
$$

where $n$ and $\Delta \tau$ are the number of delay steps and the step size, which is $1 / 150$ ps (corresponding to the $2 \mu \mathrm{m}$ 
step size of the motorized stage) in our experiment. By applying Eq. (4) to Fig. 2(a), we find that $\tau_{p}=0.58$ ps.

Once the pulse duration has been obtained from the FRAC signals, we can perform the least-squares fit on the FRAC signal envelopes with $\alpha$ and $\sigma$ as free parameters. In addition, we have included one more free parameter for the fitting to horizontally translate the calculated envelopes to adjust "time zero." Although the data quality is rather limited, as we see in Fig. 2, we have found that the fitting works reasonably well, and we have obtained the fitted values of $\alpha \sim 0 \%$ and $\sigma=1.31 \%$. The calculated envelopes using those parameters are shown in Fig. 2(a) by blue lines. To check the robustness of the fitted values, we have tried a few different initial values for $\alpha$ and $\sigma$, and found that the fitted values are very similar: $\alpha$ is always very small and $\sigma$ is always around $1.3 \%-1.4 \%$. Note that $1.3 \%$ wavelength stability at $12 \mu \mathrm{m}$ means that the central wavelength of KU-FEL may be off by $\pm 0.155 \mu \mathrm{m}$ (for so-called \pm one-sigma), while the spectral width of KU-FEL is $\sim 0.25 \mu \mathrm{m}$ (FWHM).

To compare with the above results, we have carried out two separate measurements. The first one is an IAC measurement, and we have found the autocorrelation width of 0.9 ps [Fig. 3(a)], which means $\tau_{p}=$ $0.64 \mathrm{ps}$ if we assume a Gaussian temporal shape. The second one is a single-shot measurement of the spectra of temporally selected FEL micropulses at $11 \mu \mathrm{m}$ through sum-frequency mixing (SFM) by introducing an external Nd:YAG laser [9]. In Fig. 3(b) we present the central wavelength distribution of the temporally selected micropulses by the 20 ns time window, which has been set at the peak of the macropulse envelope. This shows that the wavelength stability of the temporally selected micropulses is about $0.3 \%$ (root-mean-square). The results we obtained for the pulse duration using the IAC measurement and for the wavelength stability using the SFM measurement turned out to be different from those obtained by the FRAC measurement. The reason for this can be that the operation conditions of FEL were somehow different, since the different experiments were carried out on different days. Besides, we point out that the wavelength stability using the SFM measurement is regarding the temporally selected micropulses located around the peak of the macropulse envelope where the saturation of amplification is reached. It is reasonable that the wavelength appears more stable for such micropulses.

In conclusion, we have reported the pulse duration and wavelength stability measurements of a mid-IR FEL using
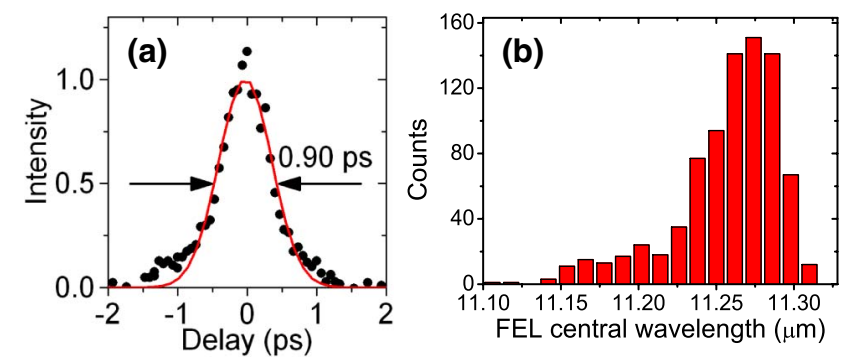

Fig. 3. (Color online) (a) IAC signals of KU-FEL at $12 \mu \mathrm{m}$ (black dots) and the fitted curve (red line) assuming a Gaussian temporal shape. (b) Distribution of the FEL central wavelength measured by the SFM technique [9] at $\sim 11 \mu \mathrm{m}$.

a FRAC method. We point out that even the pulse duration measurement using the FRAC method is not as trivial as it sounds if the amounts of chirp and wavelength stability are not known in advance. We have described a simple but, to our knowledge, new way to obtain a reliable pulse duration from the FRAC signals under such situations, and found the pulse duration of 0.58 ps without any notable chirp. After obtaining the pulse duration, we have carried out the parameter fittings, and found that the wavelength stability of KU-FEL is about $1.3 \%$.

This work was supported by a grant-in-aid for scientific research from the Ministry of Education and Science of Japan.

\section{References}

1. P. G. O'Shea and H. P. Freund, Science 292, 1853 (2001).

2. H. Ohgaki, T. Kii, K. Masuda, H. Zen, S. Sasaki, T. Shiiyama, R. Kinjo, K. Yoshikawa, and T. Yamazaki, Jpn. J. Appl. Phys. 47, 8091 (2008).

3. J. C. Tully, Science 312, 1004 (2006).

4. J. C. Diels and W. Rudolph, Ultrafast Phenomena (Academic, 1996).

5. J. Xu, G. M. H. Knippels, D. Oepts, and A. F. G. van der Meer, Opt. Commun. 197, 379 (2001).

6. H. Schneider, O. Drachenko, S. Winnerl, M. Helm, and M. Walther, Appl. Phys. Lett. 89, 133508 (2006).

7. H. Schneider, O. Drachenko, S. Winnerl, M. Helm, T. Maier, and M. Walther, Infrared Phys. Technol. 50, 95 (2007).

8. Y. Qin, T. Nakajima, T. Kii, and H. Ohgaki, Jpn. J. Appl. Phys. 51, 102704 (2012).

9. X. Wang, T. Nakajima, H. Zen, T. Kii, and H. Ohgaki, Opt. Lett. 37, 5148 (2012). 